\title{
Modeling and Simulation of Oxygen Precipitation in CZ Silicon
}

\author{
B. G. Ko, S. H. Yoon ${ }^{a}$, and K. D. Kwack \\ Department of Electronic Engineering, Hanyang University \\ Seoul 133-791, Korea \\ ${ }^{a}$ Electronic Department, Doowon Institute of Technology \\ Juksan, Ansung 456-890, Korea
}

\begin{abstract}
This paper describes modeling and simulation for the growth and dissolution of oxygen precipitates in Czochralski silicon(CZ) wafers during heat treatment. Growth and dissolution rates are newly derived and inserted into a set of chemical rate equations(CREs) and a Fokker-Planck equation(FPE). Annealing ambients and surface conditions are taken into account for solving continuity equations in order to calculate oxygen depth profile and oxygen precipitates density more precisely.
\end{abstract}

\section{Introduction}

Intersitial oxygen in silicon crystal has important roles for VLSI/ULSI fabrications. Supersaturated oxygen gives rise to produce defects such as oxygen precipitates in silicon bulk. One of the best of silicon wafers for ULSI is thought to have no defects in device layer $(\sim 10 \mu \mathrm{m}$ thick), so called denuded zone, and adequate oxygen precipitates in the bulk region. If too much oxyge precipitates are generated, interstitial oxygen concentrations are so low level that weaken the strength of silicon matrix. And oxygen precipitates act as gettering sites for unwanted metallic contaminants. So interstitial oxygen concentrations and densities must be controlled to a optimum level. To do this, understanding oxygen precipitation mechanism and designing thermal processes are extremely important.

In this work, the growth and dissolution rates were derived with matrix concentrations based on the diffusion-limited growth law and detailed balance equilibrium theory [1]. And, continuity equations for interstitial oxygen, silicon interstitials and vacancies were solved considering annealing ambients and surface conditions. Generally, before thermal process, in oredr to protect the surface of silicon thin oxide films are coated. So, boundary conditions in the continuity equations during thermal processes are considered as the interface of $\mathrm{SiO}_{2} / \mathrm{Si}$. In oxidizing ambient, surface oxygen solubility is enhanced 3-4 times than the normal solubility [2] and at the interface of $\mathrm{SiO}_{2} / \mathrm{Si}$ self interstitials are injected [3]. Therefore, in oxidizing ambient, interstitial injection depending on the growth rate of oxide film, surface recombination, and oxygen solubility enhancement effects must be included. 


\section{Modeling growth and dissolution rates of oxygen precipitates}

Solving the diffusion equation in the spherical coordinates outside a precipitate containing $n$ oxygen atoms gives

$$
\frac{\partial n}{\partial t}=\frac{\partial n}{\partial r} \frac{\partial r}{\partial t}=\frac{\partial n}{\partial r} \frac{V_{S i O_{2}}}{2} \frac{D_{O}}{r}\left(C_{O}-C_{O}^{t f}\right)=4 \pi r\left(\frac{1+e_{T}}{1+e_{c}}\right)^{3} D_{O}\left(C_{O}-C_{O}^{t f}\right),
$$

where $C_{O}$ is the concentration of interstitial oxygen, $C_{O}^{i f}$ is the concentration of a precipitation-matrix interface, $D_{O}$ is the diffusion coefficient of oxygen atom in silicon, $e_{\mathfrak{c}}$ is residual strain of a constrained precipitate, $e_{T}$ is the linear misfit and $V_{\mathrm{S}_{1} \mathrm{O}_{2}}$ is the volume of a $\mathrm{SiO}_{2}$. We can write the growth law of a precipitate taking into account the activation probability $\exp \left(\Delta G_{a} / k T\right)$, where $\Delta G_{a}$ is the activation free energy barrier, as follow.

$$
\frac{\partial n}{\partial t}=4 \pi r\left(\frac{1+e_{T}}{1+c_{c}}\right)^{3} D_{o}\left(C_{O}-C_{o}^{i f}\right) \exp \left(-\frac{\Delta G_{a}}{k T}\right)=g_{n}-d_{n+1} .
$$

Therefore, as the first term in the above equation, growth rate $g_{n}$ can be given as

$$
g_{n}=4 \pi r\left(\frac{1+e_{T}}{1+e_{c}}\right)^{3} D_{O} C_{O} \exp \left(-\frac{\Delta G_{a}}{k T}\right) \text {. }
$$

By the principle of detailed balance in thermal equilibrium, we can relate the dissolution rate to the growth rate. In thermal equilibrium, the time-variation of the size distribution of precipitation must be zero, which can be satisfied only if $g_{n} / d_{n+1}=\exp (-\Delta G / k T)$. Therefore, dissolution rate $d_{n+1}$ can be written as

$$
d_{n+1}=4 \pi r\left(\frac{1+e_{T}}{1+e_{c}}\right)^{3} D_{O} C_{o}^{*} \exp \left(-\frac{\Delta G_{a}-\Delta G}{k T}\right),
$$

where $\Delta G$ is the Gibbs' free energy difference between $(n+1)$ and $(n)$ state. In the above equation, the thermal equilibrium concentrarion $C_{o}^{*}$ must be changed by effective equilibrium value $C_{O e f f}^{*}$ including detailed equlibrium conditions. $C_{O \text { eff }}^{*}$ can be calculated by setting $\partial G / \partial r=0$ and solving for $C_{O}=C_{D \text { esf }}^{*}$ as follow,

$$
C_{O e f f}^{*}=C_{O}^{*}\left(\frac{C_{I}}{C_{i}^{*}}\right)^{\gamma_{I}}\left(\frac{C_{V}}{C_{V}^{*}}\right)^{\gamma_{\nu}} \exp \left[V_{O}\left(2 \alpha / r+6 \mu_{s i} e_{T} e_{\mathrm{c}}\right) / k T\left(\frac{1+e_{T}}{1+e_{c}}\right)^{3}\right],
$$

where $C_{1}$ and $C_{V}$ denote the concentration of silicon interstitial and vacancy, respectively, $C_{I}^{*}$ and $C_{V}^{*}$ are their thermal equilibrium values, $V_{O}=V_{S_{2} O_{2}} / 2, \alpha$ is the interfacial energy per unit area of the precipitate, $\mu_{s i}$ is the shear modulus of silicon. In the case of $C_{O}=C_{O}^{*}, C_{I}=C_{J}^{*}, C_{V}^{*}=C_{V}^{*}, \alpha=0$, and $e_{c}=0$, equation (5) satisfies the detailed balance condition. The induced growth and dissolution rates are inserted into the FPE combined with CREs and numerically solved. The detailed expressions of unexplained terms in above equations and numerical methods can be found in $[4][5][6]$.

\section{Boundary conditions for continuity equations}

Continuity equations for interstitial oxygen, silicon interstitials and vacancies are solved considering annealing ambients and surface conditions. We assume that the 
concentration of interstitial oxygen at the surface is retaining thermal equilibrium value during all the time of thermal processes at inert conditions. But in the case of oxygen ambient, oxygen solubility is enhanced depending on oxidation conditions. And we have considered the following generation and recombination of point defects. We assume the injection rate of the interstitial, $G$, to be proportional to the oxidation rate,

$$
G=\Theta C_{s i} v_{o x}\left(\frac{v_{o x}}{v_{\text {ini }}}\right)^{g p o w},
$$

where $\Theta$ is an empirical constant, $C_{s i}$ is the concentration of atoms in the silicon lattice, $v_{i n i}$ is the thin regime oxidation rate, and gpow is an empirical constant. Parts of the injected interstitials will be captured at kinks at the silicon surface. We assume that the surface recombination rate $R_{\text {reg }}^{I}$ is proportional to the number of excess interstitials,

$$
R_{\text {reg }}^{I}=c_{1}\left(C_{I}-C_{I}^{*}\right),
$$

where $c_{1}$ is the surface recombination coefficient of interstitials. The surface recombination coefficient is assumed to be dependent on the movement of the silicon-oxide interface due to oxidation:

$$
c_{1}=K_{\text {surf }}\left[K_{\text {rat }}\left(\frac{v_{\text {ox }}}{v_{\text {ini }}}\right)^{\text {Kpow }}+1\right],
$$

where $K_{\text {surf }}, K_{\text {rat }}$, and $K_{\text {pow }}$ are empirical constants. Equations (6) -(8) are empirical and also used in SUPREM-IV. For the vacancies we assume a similar behavior as described in equation (7) and represented as $R_{r e g}^{V}=c_{2}\left(C_{V}-C_{V}^{*}\right)$, where $c_{2}$ is the surface recombination coefficient of vacancies. We disregard any dependence of $c_{2}$ on the oxidation rate. As a result, the normal flux of point defects at the $\mathrm{Si}_{-} \mathrm{SiO}_{2}$ interface are

$$
\begin{aligned}
\mathrm{J}_{\mathrm{I}} \cdot \mathbf{n} & =c_{1}\left(C_{I}-C_{I}^{*}\right)-G \\
\mathrm{~J}_{\mathrm{V}} \cdot \mathbf{n} & =c_{2}\left(C_{V}-C_{V}^{*}\right) .
\end{aligned}
$$

\section{Results and discussion}

Figs. 1 and 3 show the simulated results of precipitates size distributions as a function of the depth after the HI-LO-HI annealings [7]. At the surface region, denuded zones were formed. From the experimental data of Figs. 2 and 4, it can be seen that surface oxygen concentrations after final anneal steps are considerably greater than the normal equilibrium concentrations at that temperatures. This results are caused by the enhanced solubility of oxygen during oxidation. Other simulation results are not matched with experimental results at the vicinity of surface. [6] On the other hand, our simulation results are agreed very well at both surface region and precipitated bulk region with experimental ones. And in the $\mathrm{N}_{2}$ condition, residual interstitial oxygen concentrations in the bulk region are reduced as shown in Fig. 2. From this result, it is confirmed that oxygen precipitation is retarded in oxidizing annealing ambients.

\section{References}

[1] C. Kittel and H. Kroemer, Thermal Physics, 2nd ed. (W. H. Freeman and Company, New York), p. 407, 1980. 
[2] J. C. Mikkelsen, jr., Appl. Phys. Lett., vol. 41, no. 2. pp. 871-873, 1982.

[3] S. T. Dunham, J. Appl. Phys., vol. 71, no. 1, pp. 685-696, 1992.

[4] M. Schrems, in Oxygen in silicon, Semiconductor and Semimetals, edited by F. Shimura(Academic Press, New York, 1994), vol. 42.

[5] S. Senkader, et al., J. Appl. Phys., vol. 78, no. 11, pp. 6469-6476, 1995.

[6] J. Easfandiary, et al., J. Electrochem. Soc., vol. 143, no. 3; pp. 995-1001, 1996.

[7] S. Isomae, et al., J. Appl. Phys., vol. 55, no. 4, pp. 817-824, 1984.

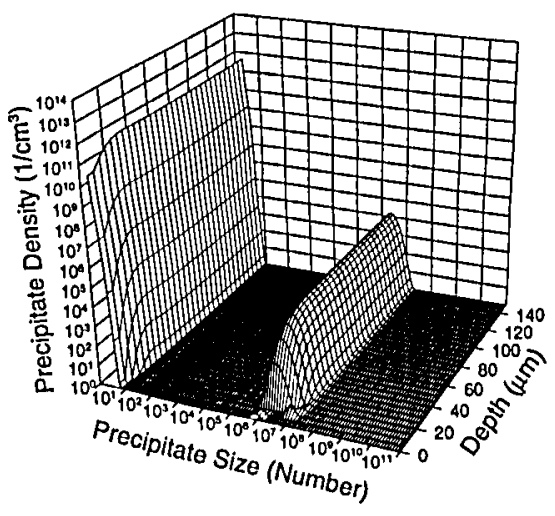

Figure 1: Calculated size distribution of oxygen precipitates versus depth after a HI-LO-HI anneal, $1100^{\circ} \mathrm{C}(3 \mathrm{~h}$, $\left.\mathrm{N}_{2}\right)+650^{\circ} \mathrm{C}\left(16 \mathrm{~h}, \mathrm{~N}_{2}\right)+1000^{\circ} \mathrm{C}\left(4 \mathrm{~h}, \mathrm{O}_{2}\right)$.

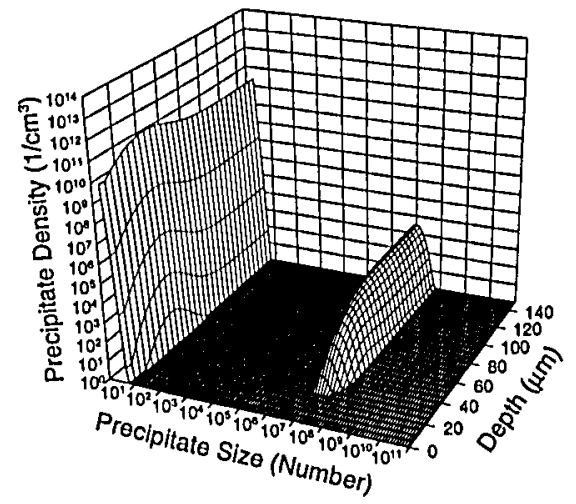

Figure 3: Calculated size distribution of oxygen precipitates versus depth after a HI-LO-HI anneal, $1100^{\circ} \mathrm{C}(16 \mathrm{~h}$, $\left.\mathrm{N}_{2}\right)+650^{\circ} \mathrm{C}\left(16 \mathrm{~h}, \mathrm{~N}_{2}\right)+1000^{\circ} \mathrm{C}\left(16 \mathrm{~h}, \mathrm{O}_{2}\right)$.

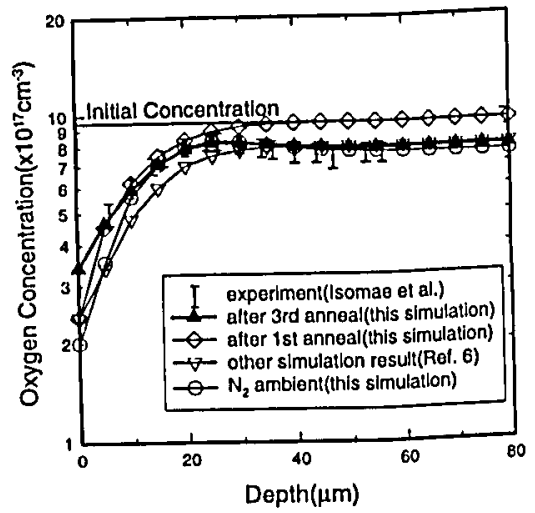

Figure 2: Depth profiles of the Interstitial oxygen concentration during each step of an $1100^{\circ} \mathrm{C}\left(3 \mathrm{~h}, \mathrm{~N}_{2}\right)+650^{\circ} \mathrm{C}(16 \mathrm{~h}$, $\left.\mathrm{N}_{2}\right)+1000^{\circ} \mathrm{C}\left(4 \mathrm{~h}, \mathrm{O}_{2}\right)$ anneal.

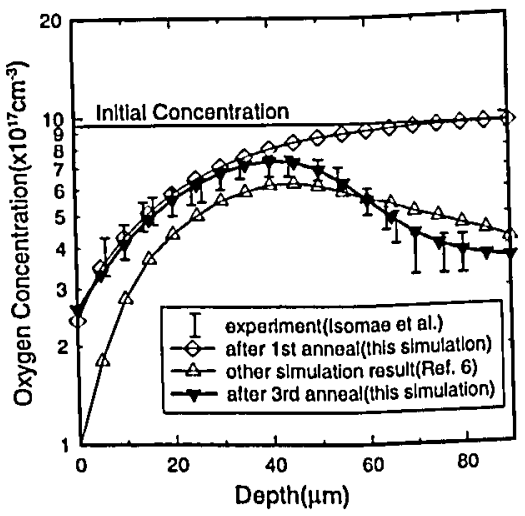

Figure 4: Depth profiles of the Interstitial oxygen concentration during each step of an $1100^{\circ} \mathrm{C}\left(16 \mathrm{~h}, \mathrm{~N}_{2}\right)+650^{\circ} \mathrm{C}$ $\left(16 \mathrm{~h}, \mathrm{~N}_{2}\right)+1000^{\circ} \mathrm{C}\left(16 \mathrm{~h}, \mathrm{O}_{2}\right)$ anneal. 